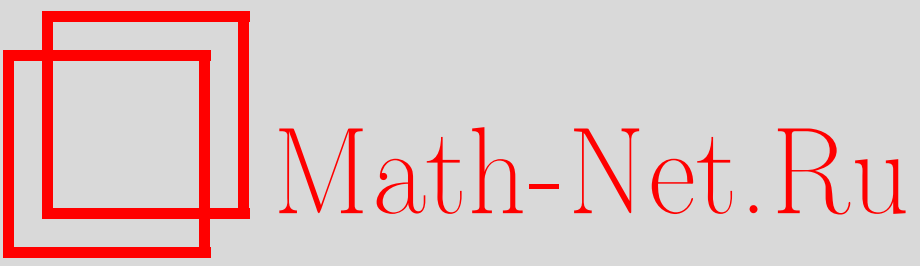

А. П. Ильин, Аналитическое представление интеграла перекрытия трех волновых функций рассеяния ядер в кулоновском поле, ТМФ, 2006, том 146, номер 2, 311-328

DOI: https://doi.org/10.4213/tmf2037

Использование Общероссийского математического портала Math-Net.Ru подразумевает, что вы прочитали и согласны с пользовательским соглашением

http://www . mathnet.ru/rus/agreement

Параметры загрузки:

IP: 54.162 .127 .20

26 апреля 2023 г., 14:59:28 
ТЕОРЕТИЧЕСКАЯ

И МАТЕМАТИЧЕСКАЯ

ФИЗИКА

Том 146, № 2

февраль, 2006

$\begin{array}{ll}\text { (c) } 2006 \text { г. } & \text { А. П. Ильин* }\end{array}$

\title{
АНАЛИТИЧЕСКОЕ ПРЕДСТАВЛЕНИЕ ИНТЕГРАЛА ПЕРЕКРЫТИЯ ТРЕХ ВОЛНОВЫХ ФУНКЦИЙ РАССЕЯНИЯ ЯДЕР В КУЛОНОВСКОМ ПОЛЕ
}

\begin{abstract}
На основе формализма интегральных представлений функций гипергеометрического типа найдены два способа описания кулоновского развала легких слабосвязанных атомных ядер на тяжелых многозарядных ядрах. В первом способе интеграл перекрытия, содержащий произведение трех волновых функций рассеяния ядер в кулоновском поле, разлагается в двукратный ряд гипергеометрического типа по произведениям двух гипергеометрических функций Гаусса. Ряд является сходящимся, по меньшей мере, при выполнении трехчастичных кинематических соотношений. Во втором способе тот же интеграл перекрытия приводится к однократному контурному интегралу, содержащему гипергеометрическую функцию Гаусса и две дополнительные биномиальные функции.
\end{abstract}

Ключевые слова: кулоновский развал, интегральные представления, контурные интегралы, гипергеометрические функции.

\section{1. ВВЕДЕНИЕ}

Для интерпретации развала слабосвязанных двухкластерных ядер при рассеянии на тяжелых ядрах к настоящему времени разработано множество методов, имеющих различные области применения, отличающихся степенью точности модели и соответственно качеством согласия с экспериментом.

Для описания квазисвободного развала применяли ораque-модель [1], разработанную на основе геометрической модели Сербера [2], и упрощенную форму метода искаженных волн [3]. Дифракционная модель с учетом кулоновского взаимодействия [4] широко применялась для описания различных ядерных реакций, индуцированных легкими ионами при средних энергиях рассеяния.

Для интерпретации экспериментов по развалу обычно используется борновское приближение (БП) метода искаженных волн (МИВ). По способу выбора взаимодействия в матричном элементе развала и систем координат Якоби МИВ классифицируется на prior-форму метода Остерна [5], [6] и метод Баура-Траутмана. Последний подразделяется на prior-форму и post-форму [6], [7].

\footnotetext{
*Институт ядерных исследований НАН Украины, Киев, Украина. E-mail: ilyin@kinr.kiev.ua
} 
В работе [5] метод Остерна был применен к развалу дейтронов при $E_{d}=12 \mathrm{MэB}$ на ядрах ${ }^{198} \mathrm{Au}$. Хорошего описания корреляционных энергетических спектров не было получено. Проведенный в работе анализ показал важную роль кулоновского развала и необходимость учета тех эффектов, которые позже были учтены при использовании post-формы МИВ в работе [7]. В этой работе для расчета матричных элементов развала дейтронов был использован метод исключения амплитуды кулоновского развала, вычисленной в работе [8] в приближении нулевого радиуса взаимодействия.

Лучшее согласие с эспериментом при использовании метода Остерна было получено в работе [9] при описании развала ${ }^{3} \mathrm{He} \mathrm{c} \mathrm{энергией} 90$ МэВ на ядрах средней массы. В этом случае роль кулоновского развала невелика. Дальнейшим развитием этого подхода является метод связанных каналов с дискретизацией континуума (СКДК) [10]. На основе этого метода выполнена интерпретация [11] экспериментов по рассеянию и развалу дейтронов и ${ }^{3}$ Не при энергиях выше кулоновского барьера, а также ядер ${ }^{6} \mathrm{Li},{ }^{7} \mathrm{Li}$ и ${ }^{12} \mathrm{C}$. Однако такие расчеты являются длительными и требуют больших ресурсов.

Нужно отметить, что приближение МИВ, основанное на prior-форме метода Баура-Траутмана, дает при описании [6] развала ядер ${ }^{6} \mathrm{Li}$ на ядрах ${ }^{208} \mathrm{~Pb}$ согласие результатов с экспериментом не хуже, чем метод СКДК. Еще ранее post-форма указанного метода, реализованная в программе BREAK4, применялась для интерпретации развала гелионов ${ }^{3} \mathrm{He}$ на ядрах ${ }^{90} \mathrm{Zr}$ [12]. В указанной программе для интегрирования по асимптотической области используется метод Винсента-Форчуна [13]. В этом методе число угловых моментов несвязанных состояний, в которых может оказаться одна из частиц, должно быть ограниченным. В программе BREAK4 оно равно 15. Если для системы ${ }^{3} \mathrm{He}=d+p$, энергия связи которой 5.5 МэВ, такое число подходит, то для более слабосвязанных ядер согласно оценкам, использующим результаты адиабатической теории [14], оно дожно быть значительно больше. Вследствие этого отодвигается нижняя граница асимптотической области для кулоновских волновых функций [15], начиная с которой применяется метод Винсента-Форчуна, и значительно возрастает число парциальных компонентов матрицы реакции развала в четырехкратной сумме по трем угловым моментам частиц и одной проекции углового момента.

Вышеуказанную трудность можно обойти путем выделения кулоновского развала на две заряженные частицы в отдельное слагаемое в выражении матричного элемента развала.

В работе [16] была выполнена асимптотическая оценка влияния трехчастичных кулоновских эффектов на развал легкого иона в кулоновском поле многозарядного тяжелого ядра. Расчеты показали, что такие эффекты имеют существенное значение для развала ядер ${ }^{6} \mathrm{Li},{ }^{7} \mathrm{Li}$ только тогда, когда энергия относительного движения частиц из развала $\epsilon_{k}<50$ кэВ. В эксперименте очень трудно выделить область сверхнизких энергий по $\epsilon_{k}$. Поэтому в данной работе вкладом указанных эффектов мы пренебрегаем.

В разделе 2 на основе известных выражений определяется матричный элемент 
кулоновского развала в виде производной от интеграла перекрытия. Последний представляется через двукратный контурный интеграл. Его выражение в разделах 3 и 4 используется для получения двух различных формул. Все проведенные выкладки были затем проверены с помощью пакета Mathematica 5. В разделе 5 обсуждается возможность учета поправок, связанных с конечностью радиуса взаимодействия между кластерами.

\section{2. ПРЕДСТАВЛЕНИЕ КУЛОНОВСКОГО ИНТЕГРАЛА В ВИДЕ ДВУКРАТНОГО КОНТУРНОГО ИНТЕГРАЛА}

Интеграл перекрытия, содержащий произведение трех волновых функций рассеяния частиц в кулоновском поле, появляется в задаче о развале слабосвязанных легких атомных ядер при рассеянии на тяжелых ядрах, если использовать при определении матрицы перехода в еe post-форме БП искаженных волн и приближение псевдопотенциала нулевого радиуса для ядерного взаимодействия между кластерами, на которые распадается слабосвязанное ядро. Тогда матричный элемент перехода представляется интегралом

$$
T_{f i(\omega)}=D_{b a} \iint e^{-\omega R} \Psi^{-}\left(\vec{k}_{a}, \vec{r}_{a}\right)^{*} \Psi^{-}\left(\vec{q}_{b}, \vec{R}_{b}\right)^{*} \delta(\vec{r}) \Psi^{+}\left(\vec{q}_{c}, \vec{R}\right) d^{3} r d^{3} R .
$$

Для кластеров $a$ и $b$, входящих в ядро $c$, константа связи $D_{b a}<0$. Чтобы определить обобщенное значение [17] этого интеграла, здесь введен регуляризующий экспоненциально спадающий множитель, как это обычно делается для определения матрицы перехода в post-форме [13]. В отличие от других работ, мы не будем переходить к пределу $\omega \rightarrow 0$, однако будем полагать $\omega$ настолько малым, что можно вычислить интеграл с практически необходимой точностью. Такой подход дает возможость при численных расчетах правильно определить показатели комплексных экспонент. Пространственные координаты частиц записаны через относительные координаты Якоби: для входного канала $\{\vec{r}, \vec{R}\}$, где $\vec{r}=\vec{r}_{b}-\vec{r}_{a}, \vec{R}=-\vec{R}_{t}$, и для выходного канала $\left\{\vec{r}_{a}, \vec{R}_{b}\right\}$ или $\left\{\vec{r}_{b}, \vec{R}_{a}\right\}$. Размерности выбираем так, что $\hbar=c=1$.

В приближении нулевого радиуса взаимодействия между кластерами $b$ и $a$, на которые распадается легкое ядро $c$ при рассеянии на тяжелом ядре $t$, пренебрегаем кулоновским взаимодействием кластеров между собой. В приближении искаженных волн пренебрегаем также добавкой поляризующего кулоновского потенциала $V_{b}\left(r_{b}\right)-V_{b}\left(R_{b}\right)$ к потенциалу нулевого радиуса взаимодействия в выражении для матричного элемента.

Волновая функция частицы $c$ выражается [18] через вырожденную гипергеометрическую функцию [19], [20] Куммера:

$$
\Psi^{+}\left(\vec{q}_{c}, \vec{R}\right)=A\left(\eta_{c}\right) e^{i\left(\vec{q}_{c} \vec{R}\right)} \Phi\left(-i \eta_{c}, 1 ; i z_{c}(\vec{R})\right) .
$$

Здесь введены следующие обозначения:

$$
A\left(\eta_{c}\right)=e^{-\pi \eta_{c} / 2} \Gamma\left(1+i \eta_{c}\right), \quad z_{c}(\vec{R})=q_{c} R-\left(\vec{q}_{c} \vec{R}\right), \quad \eta_{c}=\frac{M_{c} M_{t} e^{2}\left(Z_{a}+Z_{b}\right) Z_{t}}{\left(M_{c}+M_{t}\right) q_{c}} .
$$


Волновая функция частицы $a$ в выходном канале реакции асимптотически представляется в виде суммы падающей кулоновской плоской волны и сходящейся сферической волны, искаженной кулоновским полем. Ее комплексно-сопряженная форма имеет вид

$$
\Psi^{-}\left(\vec{k}_{a}, \vec{r}_{a}\right)^{*}=A\left(\eta_{a}\right) e^{-i\left(\vec{k}_{a} \vec{r}_{a}\right)} \Phi\left(-i \eta_{a}, 1 ; i \tilde{z}_{a}\left(\vec{r}_{a}\right)\right),
$$

параметры определяются следующим образом:

$$
A\left(\eta_{a}\right)=e^{-\pi \eta_{a} / 2} \Gamma\left(1+i \eta_{a}\right), \quad \tilde{z}_{a}\left(\vec{r}_{a}\right)=k_{a} r_{a}+\left(\vec{k}_{a} \vec{r}_{a}\right), \quad \eta_{a}=\frac{M_{a} M_{t} e^{2} Z_{a} Z_{t}}{\left(M_{a}+M_{t}\right) k_{a}} .
$$

Аналогичный вид имеет комплексно-сопряженная волновая функция частицы $b$ c той разницей, что вектор $\vec{R}_{b}$ определяет положение частицы $b$ относительно центра масс частицы $a$ и ядра-мишени $t$, а вектор $\overrightarrow{q_{b}}$ есть импульс, сопряженный этой координате Якоби. Следовательно, $\overrightarrow{q_{b}}$ есть импульс частицы $b$ в системе центра масс трех частиц:

$$
\Psi^{-}\left(\vec{q}_{b}, \vec{R}_{b}\right)^{*}=A\left(\eta_{b}\right) e^{-i\left(\vec{q}_{b} \vec{R}_{b}\right)} \Phi\left(-i \eta_{b}, 1 ; i \tilde{z}_{b}\left(\vec{R}_{b}\right)\right) ;
$$

параметры определяются соотношениями

$$
A\left(\eta_{b}\right)=e^{-\pi \eta_{b} / 2} \Gamma\left(1+i \eta_{b}\right), \quad \tilde{z}_{b}\left(\vec{R}_{b}\right)=q_{b} R_{b}+\left(\vec{q}_{b} \vec{R}_{b}\right), \quad \eta_{b}=\frac{M_{b} M_{t} e^{2} Z_{b} Z_{t}}{\left(M_{a}+M_{b}+M_{t}\right) q_{b}}
$$

Формула для параметра $\eta_{b}$ учитывает, что при замене

$$
r_{b} \rightarrow\left(\left|\frac{\left(M_{a}+M_{t}\right) \vec{R}_{b}}{M_{t}}-\frac{M_{a} \vec{r}}{M_{t}}\right|\right)_{\vec{r}=0}
$$

выражение для кулоновского потенциала в уравнении для функции $\Psi^{-}\left(\vec{q}_{b}, \vec{R}_{b}\right)^{*}$ перенормируется на коэффициент

$$
\delta=\frac{M_{t}}{M_{a}+M_{t}}
$$

Между выбранными нами системами координат Якоби для выходного и входного каналов имеют место соотношения

$$
\vec{r}_{a}=\vec{R}-\gamma \vec{r}, \quad \vec{R}_{b}=\delta \vec{R}_{x}, \quad \vec{R}_{x}=\vec{R}+\xi \vec{r}
$$

где

$$
\gamma=\frac{M_{b}}{M_{a}+M_{b}}, \quad \xi=\frac{M_{a}\left(M_{a}+M_{b}+M_{t}\right)}{\left(M_{a}+M_{b}\right) M_{t}} .
$$

После замены переменной $\vec{R}_{b}=\delta \vec{R}_{x}$ уравнение для функции Куммера, фигурирующей в выражении (6), записывается в виде

$$
\left(\Delta_{R_{x}}-2 i\left(\vec{p}_{b} \cdot \vec{\nabla}_{R_{x}}\right)-\frac{2 \eta_{b} p_{b}}{R_{x}}\right) \Phi\left(-i \eta_{b}, 1 ; i \tilde{z}_{x}\left(\vec{R}_{x}\right)\right)=0
$$

где

$$
\vec{p}_{b}=\delta \vec{q}_{b}, \quad \tilde{z}_{x}\left(\vec{R}_{x}\right)=p_{b} R_{x}+\left(\vec{p}_{b} \vec{R}_{x}\right), \quad \eta_{b}=\frac{M_{b} M_{t}^{2} e^{2} Z_{b} Z_{t}}{\left(M_{a}+M_{t}\right)\left(M_{a}+M_{b}+M_{t}\right) p_{b}} .
$$


Подставляем выражения (2), (4), (6) для волновых функций в правую часть (1) и, учитывая замены (10)-(12), находим

$$
\begin{aligned}
T_{f i(\omega)} & =D_{b a} A\left(\eta_{a}, \eta_{b}, \eta_{c}\right) \iint \exp \left(i\left[\left(\vec{q}_{c} \vec{R}\right)-\left(\vec{k}_{a} \vec{r}_{a}\right)-\left(\vec{q}_{b} \vec{R}_{b}\right)\right]-\omega R\right) \times \\
& \times \Phi\left(-i \eta_{a}, 1 ; i \tilde{z}_{a}\left(\vec{r}_{a}\right)\right) \Phi\left(-i \eta_{b}, 1 ; i \tilde{z}_{x}\left(\vec{R}_{x}\right)\right) \delta(\vec{r}) \Phi\left(-i \eta_{c}, 1 ; i z_{c}(\vec{R})\right) d^{3} r d^{3} R
\end{aligned}
$$

где

$$
A\left(\eta_{a}, \eta_{b}, \eta_{c}\right)=A\left(\eta_{a}\right) A\left(\eta_{b}\right) A\left(\eta_{c}\right) .
$$

Имеют место соотношения, связывающие в различных системах координат Якоби суммы скалярных произведений импульсов частиц из канала развала на сопряженные им координаты:

$$
\left(\vec{k}_{a} \vec{r}_{a}\right)+\left(\vec{q}_{b} \vec{R}_{b}\right)=\left(\vec{k}_{b} \vec{r}_{b}\right)+\left(\vec{q}_{a} \vec{R}_{a}\right)=(\vec{k} \vec{r})+\left(\vec{q}_{f} \vec{R}\right),
$$

где введены следующие обозначения:

$$
\vec{q}_{f}=\vec{q}_{a}+\vec{q}_{b}, \quad \vec{q}=\vec{q}_{c}-\vec{q}_{f}, \quad \vec{k}=\frac{M_{a} \vec{q}_{b}-M_{b} \vec{q}_{a}}{M_{a}+M_{b}} .
$$

Подставляя в выражение (13) последнее из равенств (14) и учитывая соотношения $(10)$ и (15), в результате интегрирования по пространству переменной $\vec{r}$ получаем интеграл по объему, определяющий матричный элемент перехода в приближении нулевого радиуса. Затем, используя дифференцирование по параметру $\omega$ (что можно сделать, поскольку интеграл сходится абсолютно), представляем его в следующей форме:

$$
\begin{aligned}
T_{f i(\omega)}= & -D_{b a} A\left(\eta_{a}, \eta_{b}, \eta_{c}\right) \frac{\partial X_{0, \omega}}{\partial \omega}, \\
X_{0, \omega}=\int & \frac{1}{R} e^{-\omega R+i(\vec{q} \vec{R})} \Phi\left(-i \eta_{a}, 1 ; i \tilde{z}_{a}(\vec{R})\right) \times \\
& \times \Phi\left(-i \eta_{b}, 1 ; i \tilde{z}_{x}(\vec{R})\right) \Phi\left(-i \eta_{c}, 1 ; i z_{c}(\vec{R})\right) d^{3} R .
\end{aligned}
$$

Здесь в подынтегральное выражение входят три вырожденные гипергеометрические функции Куммера [19], [20] с однотипными параметрами и аргументами. Для их дальнейшего преобразования будем использовать представление этих функций через контурный интеграл [18], [20]. Из формулы (6.11 (2)) книги [20] следует соотношение

$$
\Phi(-i \eta, 1 ; i z)=-\frac{1}{2 \pi i} \int_{1}^{(0+)} e^{i z t}(-t)^{-i \eta-1}(1-t)^{i \eta} d t .
$$

Требование $\operatorname{Re}(1+i \eta)>0$, очевидно, выполняется. Все степени берутся в смысле главного значения, т.е. с наименьшим по абсолютной величине значением аргумента основания степени. Контур интегрирования начинается в точке $t=1$ и обходит точку $t=0$ в положительном направлении.

В результате подстановки в выражение (17) вместо функций Куммера их интегральных представлений и перестановки порядка интегрирований (четырехкратный 
интеграл сходится, поскольку $\omega>0)$ получаем следующее представление для интеграла перекрытия:

$$
\begin{aligned}
X_{0, \omega}= & -\frac{1}{(2 \pi i)^{3}} \int_{1}^{(0+)}(-s)^{-i \eta_{b}-1}(1-s)^{i \eta_{b}} \times \\
& \times \int_{1}^{(0+)}(-t)^{-i \eta_{a}-1}(1-t)^{i \eta_{a}} \int_{1}^{(0+)}(-\tau)^{-i \eta_{c}-1}(1-\tau)^{i \eta_{c}} \times \\
& \times \int \frac{1}{R} \exp \left(-\omega R+i\left[(\vec{q} \vec{R})+\tau z_{c}(\vec{R})+t \tilde{z}_{a}(\vec{R})+s \tilde{z}_{x}(\vec{R})\right]\right) d^{3} R d \tau d t d s .
\end{aligned}
$$

Учитывая соотношения (3), (5), (12), представляем показатель экспоненты в интеграле по $\vec{R}$ в виде

$$
\left.i\left((\vec{q} \vec{R})+\tau z_{c}(\vec{R})+t \tilde{z}_{a}(\vec{R})\right)+s \tilde{z}_{x}(\vec{R})\right)-\omega R=i(\vec{K} \vec{R})-p R,
$$

где

$$
p=\omega-i\left(t k_{a}+s p_{b}+\tau q_{c}\right), \quad \vec{K}=\vec{q}+t \vec{k}_{a}+s \vec{p}_{b}-\tau \vec{q}_{c}
$$

Теперь в результате вычисления интеграла по $\vec{R}$ находим

$$
\int \frac{1}{R} \exp (-p R+i(\vec{K} \vec{R})) d^{3} R=\frac{4 \pi}{K^{2}+p^{2}}
$$

Прежде чем подставить полученный результат в интегральное представление (18), преобразуем его. Учитывая соотношения (19), представим знаменатель в выражении (20) в виде

$$
K^{2}+p^{2}=U\left(\tau-\tau_{0}\right)
$$

где

$$
\begin{aligned}
U & =-2\left(\left(\vec{q} \vec{q}_{c}\right)+i \omega q_{c}+t\left(k_{a} q_{c}+\left(\vec{k}_{a} \vec{q}_{c}\right)\right)+s\left(p_{b} q_{c}+\left(\vec{p}_{b} \vec{q}_{c}\right)\right)\right), \\
-U \tau_{0} & =q^{2}+\omega^{2}-2 t s\left(k_{a} p_{b}-\left(\vec{p}_{b} \vec{k}_{a}\right)\right)+2 t\left(\left(\vec{q} \vec{k}_{a}\right)-i \omega k_{a}\right)+2 s\left(\left(\vec{q} \vec{p}_{b}\right)-i \omega p_{b}\right) .
\end{aligned}
$$

В результате подстановки правой части (21) в интеграл по переменной $\tau$ последний приобретает вид интеграла Коши:

$$
J\left(U, \tau_{0}\right)=-\frac{1}{2 \pi i} \int_{1}^{(0+)} \frac{(-\tau)^{-i \eta_{c}-1}(1-\tau)^{i \eta_{c}}}{U\left(\tau-\tau_{0}\right)} d \tau
$$

где контур интегрирования охватывает в положительном направлении разрез, соединяющий точки разветвления подынтегральной функции, но не включает внутри 
себя полюс в точке $\tau=\tau_{0}$. Поскольку этот полюс единственный на разрезанной комплексной плоскости $\tau$ (точка бесконечность - устранимая особая точка), то интеграл равен вычету в полюсе, взятому с обратным знаком, т.е.

$$
J\left(U, \tau_{0}\right)=\frac{\left(-\tau_{0}\right)^{-i \eta_{c}-1}\left(1-\tau_{0}\right)^{i \eta_{c}}}{U}=\left(-U \tau_{0}\right)^{-i \eta_{c}-1}\left(U-U \tau_{0}\right)^{i \eta_{c}}
$$

Подставляя (22) в (24), получаем

$$
J\left(U, \tau_{0}\right)=\left(a_{0}-b_{0} t-d_{0} s-h_{0} s t\right)^{-i \eta_{c}-1}\left(c_{0}-f_{0} t-g_{0} s-h_{0} s t\right)^{i \eta_{c}} .
$$

Здесь введены обозначения

$$
\begin{gathered}
a_{0}=q^{2}+\omega^{2}, \quad b_{0}=-2\left(\left(\vec{q} \vec{k}_{a}\right)-i \omega k_{a}\right), \quad d_{0}=-2\left(\left(\vec{q} \vec{p}_{b}\right)-i \omega p_{b}\right), \\
c_{0}=q^{2}+\omega^{2}-2\left(\left(\vec{q} \vec{q}_{c}\right)+i \omega q_{c}\right), \quad f_{0}=2\left(-\left(\vec{q} \vec{k}_{a}\right)+\left(\vec{k}_{a} \vec{q}_{c}\right)+k_{a} q_{c}+i \omega k_{a}\right), \\
g_{0}=2\left(-\left(\vec{q} \vec{p}_{b}\right)+\left(\vec{p}_{b} \vec{q}_{c}\right)+p_{b} q_{c}+i \omega p_{b}\right), \quad h_{0}=2\left(p_{b} k_{a}-\left(\vec{p}_{b} \vec{k}_{a}\right)\right) .
\end{gathered}
$$

Далее, подставляя $J\left(U, \tau_{0}\right)$ в интегральное представление (18) интеграла перекрытия, находим для него следующий повторный контурный интеграл:

$$
\begin{aligned}
X_{0, \omega}= & \frac{4 \pi}{(2 \pi i)^{2}} \int_{1}^{(0+)}(-s)^{-i \eta_{b}-1}(1-s)^{i \eta_{b}} \int_{1}^{(0+)}(-t)^{-i \eta_{a}-1}(1-t)^{i \eta_{a}} \times \\
& \times\left(a_{0}-b_{0} t-d_{0} s-h_{0} s t\right)^{-i \eta_{c}-1}\left(c_{0}-f_{0} t-g_{0} s-h_{0} s t\right)^{i \eta_{c}} d t d s .
\end{aligned}
$$

До сих пор мы действовали аналогично методу вычисления зоммерфельдовских интегралов для тормозного излучения при кулоновском рассеянии ядер. Для дальнейшего преобразования интеграла (27) используем разработанный автором математический прием, который можно назвать методом инфинитезимальных дробнолинейных преобразований.

\section{3. РАЗЛОЖЕНИЕ ДВУКРАТНОГО КОНТУРНОГО ИНТЕГРАЛА В ДВУКРАТНЫЙ РЯД}

Выполним в интеграле (27) дробно-линейные преобразования вида

$$
s=\frac{\rho}{\rho \alpha-\alpha+1}, \quad t=\frac{\tau}{\tau \beta-\beta+1} .
$$

Эти преобразования не изменяют контуров интегрирования и имеют по одному свободному параметру, а именно $\alpha$ и $\beta$. Находим, что сомножители в подынтеграль- 
ном выражении (27) преобразуются следующим образом:

$$
\begin{aligned}
& (1-s)^{i \eta_{b}}(-s)^{-i \eta_{b}-1} \rightarrow(1-\alpha)^{i \eta_{b}}(-\rho)^{-i \eta_{b}-1}(1-\rho)^{i \eta_{b}}(\rho \alpha-\alpha+1) \\
& (1-t)^{i \eta_{a}}(-t)^{-i \eta_{a}-1} \rightarrow(1-\beta)^{i \eta_{a}}(-\tau)^{-i \eta_{a}-1}(1-\tau)^{i \eta_{a}}(\tau \beta-\beta+1) \\
& \left(a_{0}-b_{0} t-d_{0} s-h_{0} s t\right)^{-i \eta_{c}-1} \rightarrow a_{0}{ }^{-i \eta_{c}-1}\left(\frac{\alpha \rho}{1-\alpha}+1\right)^{i \eta_{c}+1}\left(\frac{\beta \tau}{1-\beta}+1\right)^{i \eta_{c}+1} \times \\
& \quad \times\left(1-\frac{\left(b_{0}-\beta a_{0}\right)}{(1-\beta) a_{0}} \tau-\frac{\left(d_{0}-\alpha a_{0}\right)}{(1-\alpha) a_{0}} \rho-\frac{\left(-\alpha \beta a_{0}+\alpha b_{0}+\beta d_{0}+h_{0}\right)}{(1-\alpha)(1-\beta) a_{0}} \rho\right)^{-i \eta_{c}-1} ; \\
& \left(c_{0}-f_{0} t-g_{0} s-h_{0} s t\right)^{i \eta_{c}} \rightarrow c_{0} i \eta_{c}\left(\frac{\alpha \rho}{1-\alpha}+1\right)^{-i \eta_{c}}\left(\frac{\beta \tau}{1-\beta}+1\right)^{-i \eta_{c}} \times \\
& \quad \times\left(1-\frac{\left(f_{0}-\beta c_{0}\right)}{(1-\beta) c_{0}} \tau-\frac{\left(g_{0}-\alpha c_{0}\right)}{(1-\alpha) c_{0}} \rho-\frac{\left(-\alpha \beta c_{0}+\alpha f_{0}+\beta g_{0}+h_{0}\right)}{(1-\alpha)(1-\beta) c_{0}} \rho \tau\right)^{i \eta_{c}}
\end{aligned}
$$

Подставляя (29)-(31) в подынтегральное выражение в (27) и учитывая выражения для дифференциалов преобразований (28), находим, что степеннь́е функции, содержащие в основании знаменатели дробно-линейных преобразований, взаимно сокращаются.

В четвертом сомножителе в правой части формулы (31) обозначим коэффициент при $-\tau$ через $f_{1}$. Выберем далее параметры $\beta$ и $\alpha$ дробно-линейных преобразований так, чтобы коэффициент при - $\tau$ был равен нулю, а коэффициент при $\rho$ стремился К нулю при

$$
\epsilon=\alpha-\frac{g_{0}}{c_{0}} \rightarrow 0
$$

Тогда

$$
\alpha=\epsilon+\frac{g_{0}}{c_{0}} \rightarrow \frac{g_{0}}{c_{0}}, \quad \beta=\frac{f_{0}}{c_{0}}+\frac{1}{\epsilon \xi_{1}} \rightarrow \infty, \quad \xi_{1}=\frac{c_{0}^{2}}{f_{0} g_{0}+c_{0} h_{0}} .
$$

Коэффициент $f_{1}$ при $-\tau$ будет иметь предел

$$
f_{1}=\frac{f_{0}-\beta c_{0}}{(1-\beta) c_{0}} \rightarrow 1
$$

В четвертом сомножителе в (30) введем для коэффициентов следующие обозначения:

$$
\begin{gathered}
b_{1}=\frac{1-b_{0} /\left(\beta a_{0}\right)}{1-1 / \beta} \rightarrow 1, \quad d_{1}=\frac{d_{0}-\alpha a_{0}}{(1-\alpha) a_{0}} \rightarrow \frac{c_{0} d_{0}-a_{0} g_{0}}{a_{0}\left(c_{0}-g_{0}\right)}, \\
h_{1}=\frac{\alpha a_{0}-d_{0}-\left(\alpha b_{0}+h_{0}\right) / \beta}{(1-\alpha)(1-1 / \beta) a_{0}} \rightarrow \frac{\alpha a_{0}-d_{0}}{(1-\alpha) a_{0}}=-d_{1} .
\end{gathered}
$$

В этих соотношениях указаны предельные значения коэффициентов при $\epsilon \rightarrow 0$. Соотношения (34) и (35) показывают, что произведение последних сомножителей, фигурирующих в формулах (30) и (31), при $\epsilon \rightarrow 0$ дает в подынтегральном выражении множитель, содержащий добавочную сингулярность вида

$$
\left(1-f_{1} \tau+\frac{\epsilon \rho}{1-\alpha}\right)^{i \eta_{c}}\left(1-b_{1} \tau-d_{1} \rho-h_{1} \rho \tau\right)^{-i \eta_{c}-1} \rightarrow \frac{\left(1-\rho d_{1}\right)^{-i \eta_{c}-1}}{1-\tau}
$$


На первый взгляд эта сингулярность нарушает сходимость интеграла при $\tau \rightarrow 1$. Однако предельный переход $\epsilon \rightarrow 0$ следует выполнять после взятия контурных интегралов в аналитической форме.

Итак, в результате дробно-линейных преобразований приходим к следующему представлению для интеграла перекрытия (27):

$$
\begin{aligned}
X_{0, \omega}= & \frac{4 \pi}{(2 \pi i)^{2}} a_{0}^{-i \eta_{c}-1} c_{0}^{i \eta_{c}} \lim _{\epsilon \rightarrow 0}\left((1-\alpha)^{i \eta_{b}}(1-\beta)^{i \eta_{a}} \times\right. \\
& \times \int_{1}^{(0+)}(-\rho)^{-i \eta_{b}-1}(1-\rho)^{i \eta_{b}} \int_{1}^{(0+)}(-\tau)^{-i \eta_{a}-1}(1-\tau)^{i \eta_{a}} \times \\
& \left.\times\left(1-b_{1} \tau-d_{1} \rho-h_{1} \tau \rho\right)^{-i \eta_{c}-1}\left(1-f_{1} \tau+\frac{\epsilon \rho}{1-\alpha}\right)^{i \eta_{c}} d \tau d \rho\right) .
\end{aligned}
$$

Здесь в подынтегральном выражении выполним разложение степенно́й функции с показателем $i \eta_{c}$ по степеням малого при $\tau \neq 1$ параметра $\epsilon \rho /\left((1-\alpha)\left(1-f_{1} \tau\right)\right)$. Поскольку повторный контурный интеграл при конечном $\epsilon$ ограничен и подынтегральные функции, составленные из членов ряда, непрерывны на контурах интегрирования, а составленый из них ряд, очевидно, сходится равномерно для каждого заданного малого $\epsilon$, то полученный ряд можно почленно интегрировать. Тогда интегральное представление (27) приобретает вид

$$
X_{0, \omega}=4 \pi a_{0}^{-i \eta_{c}-1} c_{0}^{i \eta_{c}} \lim _{\epsilon \rightarrow 0} \sum_{m=0}^{\infty} \frac{\left(-i \eta_{c}\right)_{m}}{m !} \epsilon^{m}(1-\alpha)^{i \eta_{b}-m}(1-\beta)^{i \eta_{a}}\left(Y^{m}\right)_{\omega, \varepsilon}
$$

где

$$
\begin{aligned}
\left(Y^{m}\right)_{\omega, \varepsilon}= & \frac{1}{(2 \pi i)^{2}} \int_{1}^{(0+)}(-\rho)^{m-i \eta_{b}-1}(1-\rho)^{i \eta_{b}} \int_{1}^{(0+)}(-\tau)^{-i \eta_{a}-1}(1-\tau)^{i \eta_{a}} \times \\
& \times\left(1-f_{1} \tau\right)^{i \eta_{c}-m}\left(1-b_{1} \tau-d_{1} \rho-h_{1} \tau \rho\right)^{-i \eta_{c}-1} d \tau d \rho
\end{aligned}
$$

Рассмотрим теперь отдельно повторный интеграл (37). В этом интеграле выполним еще пару дробно-линейных преобразований с неизвестными пока параметрами $\delta$ и $\gamma:$

$$
\rho=\frac{u}{1-\delta+u \delta}, \quad \tau=\frac{v}{1-\gamma+v \gamma} .
$$

Четыре сомножителя в подынтегральном выражении преобразуются следующим образом:

$$
\begin{aligned}
& (-\rho)^{m-i \eta_{b}+1}(1-\rho)^{i \eta_{b}} \rightarrow \\
& \quad \rightarrow(1-\delta)^{i \eta_{b}-m}(-u)^{m-i \eta_{b}-1}(1-u)^{i \eta_{b}}\left(1-\frac{\delta u}{\delta-1}\right)^{-m}(1-\delta+\delta u), \\
& (-\tau)^{-i \eta_{a}-1}(1-\tau)^{i \eta_{a}} \rightarrow(1-\gamma)^{i \eta_{a}}(-v)^{-i \eta_{a}-1}(1-v)^{i \eta_{a}}(1-\gamma+\gamma v), \\
& \left(1-f_{1} \tau\right)^{i \eta_{c}-m} \rightarrow\left(1-\frac{\gamma v}{\gamma-1}\right)^{m-i \eta_{c}}\left(1-\frac{\left(\gamma-f_{1}\right) v}{\gamma-1}\right)^{i \eta_{c}-m},
\end{aligned}
$$




$$
\begin{aligned}
(1- & \left.b_{1} \tau-d_{1} \rho-h_{1} \tau \rho\right)^{-i \eta_{c}-1} \rightarrow\left(1-\frac{\gamma v}{\gamma-1}\right)^{i \eta_{c}+1}\left(1-\frac{\delta u}{\delta-1}\right)^{i \eta_{c}+1} \times \\
\times\left(1-\frac{\delta-d_{1}}{\delta-1} u+\frac{\gamma-b_{1}}{1-\gamma} v-\frac{\left(-\delta\left(\gamma-b_{1}\right)+\gamma d_{1}+h_{1}\right)}{(1-\gamma)(1-\delta)} u v\right)^{-i \eta_{c}-1} & .
\end{aligned}
$$

Параметры $\delta$ и $\gamma$ выбираем так, чтобы коэффициент при $u v$ в третьем сомножителе в (42) был равен нулю, а коэффициент в линейном по $v$ слагаемом стремился к нулю в результате предельного перехода $\lambda \rightarrow 0$. Тогда

$$
\gamma-b_{1}=\lambda \rightarrow 0, \quad \delta=\frac{\left(\lambda+b_{1}\right) d_{1}+h_{1}}{\lambda} \rightarrow \infty
$$

Затем разлагаем этот третий сомножитель по степеням малого при $u \neq 1$ параметра

$$
\frac{\lambda v}{1-\gamma}\left(1-\frac{(\delta-d) u}{\delta-1}\right)^{-1}
$$

В итоге получаем ряд вида

$$
\begin{aligned}
& \left(1-\frac{\left(\delta-d_{1}\right) u}{\delta-1}+\frac{\lambda v}{1-\gamma}\right)^{-i \eta_{c}-1}= \\
& \quad=\sum_{n=0}^{\infty} \frac{\left(i \eta_{c}+1\right)_{n}}{n !}\left(\lambda^{n}(1-\gamma)^{-n}(-v)^{n}\left(1-\frac{\left(\delta-d_{1}\right) u}{\delta-1}\right)^{-n-i \eta_{c}-1}\right) .
\end{aligned}
$$

С помощью подстановки соотношений (38)-(42) в подынтегральное выражение в (37) и замены переменных интегрирования, выполняя почленное интегрирование ряда, порожденного разложением (44), преобразуем (37) к виду

$$
\begin{aligned}
\left(Y^{m}\right)_{\omega, \epsilon}= & \lim _{\lambda \rightarrow 0}\left(\sum_{n=0}^{\infty} \frac{\left(i \eta_{c}+1\right)_{n}}{n !}\left(\lambda^{n}\right)(1-\delta)^{i \eta_{b}-m}(1-\gamma)^{i \eta_{a}-n} \times\right. \\
\times & \left(\frac{-1}{2 \pi i} \int_{1}^{(0+)}(-u)^{m-i \eta_{b}-1}(1-u)^{i \eta_{b}} \times\right. \\
& \left.\times\left(1-\frac{\left(\delta-d_{1}\right) u}{\delta-1}\right)^{-n-i \eta_{c}-1}\left(1-\frac{\delta u}{\delta-1}\right)^{i \eta_{c}-m} d u\right) \times \\
\times & \frac{-1}{2 \pi i} \int_{1}^{(0+)}(-v)^{n-i \eta_{a}-1}(1-v)^{i \eta_{a}}\left(1-\frac{\gamma v}{\gamma-1}\right)^{m} \times \\
& \left.\left.\times\left(1-\frac{\left(\gamma-f_{1}\right) v}{\gamma-1}\right)^{i \eta_{c}-m} d v\right)\right) .
\end{aligned}
$$

В силу формулы (5.8.(5)) из книги [20] справедливо следующее выражение для аналитического продолжения интегрального представления гипергеометрической функции Аппеля: при $\operatorname{Re}(c-a)>0$

$$
-\frac{1}{2 \pi i} \int_{1}^{(0+)} \frac{(-u)^{a-1}(1-u)^{-a+c-1}}{(1-y u)^{b}(1-z u)^{g}} d u=\frac{\Gamma(c-a)}{\Gamma(c) \Gamma(1-a)} F_{1}(a, b, g ; y, z) .
$$


Учитывая представление (46), полагаем в первом интеграле в формуле (45)

$$
\begin{gathered}
m-i \eta_{b}=a, \quad i \eta_{b}=-a+c-1, \quad m-i \eta_{c}=b, \\
n+1+i \eta_{c}=g, \quad y=\frac{\delta}{\delta-1}, \quad z=\frac{\delta-d_{1}}{\delta-1},
\end{gathered}
$$

а во втором интеграле

$$
\begin{gathered}
n-i \eta_{a}=a^{\prime}, \quad i \eta_{a}=-a^{\prime}+c^{\prime}-1, \quad-m=b^{\prime}, \\
m-i \eta_{c}=g^{\prime}, \quad y^{\prime}=\frac{\gamma}{\gamma-1}, \quad z^{\prime}=\frac{\gamma-f_{1}}{\gamma-1} .
\end{gathered}
$$

Отсюда находим

$$
c=m+1, \quad c^{\prime}=n+1 .
$$

После преобразования интегралов в формуле (45) к функциям Аппеля находим

$$
\begin{aligned}
& \left(Y^{m}\right)_{\omega, \epsilon}=\frac{\Gamma\left(1+i \eta_{b}\right)}{\Gamma(m+1) \Gamma\left(1-m+i \eta_{b}\right)} \sum_{n=0}^{\infty} \frac{\Gamma\left(1+i \eta_{a}\right)}{\Gamma(n+1) \Gamma\left(1-n+i \eta_{a}\right)} \times \\
& \times \frac{\left(1+i \eta_{c}\right)_{n}}{n !} \lim _{\lambda \rightarrow 0}\left((1-\delta)^{i \eta_{b}-m}(1-\gamma)^{i \eta_{a}-n} \lambda^{n} \times\right. \\
& \times F_{1}\left(m-i \eta_{b}, m-i \eta_{c}, n+1+i \eta_{c}, m+1 ; \frac{\delta}{\delta-1}, \frac{\delta-d_{1}}{\delta-1}\right) \times \\
& \left.\times F_{1}\left(n-i \eta_{a},-m, m-i \eta_{c}, n+1 ; \frac{\gamma}{\gamma-1}, \frac{\gamma-f_{1}}{\gamma-1}\right)\right) .
\end{aligned}
$$

Используя известные соотношения для Г-функций (см. [20], $(1.2(3)))$ и функций Аппеля (см. [20], $(5.11(4,1)))$, выводим

$$
\begin{aligned}
\left(Y^{m}\right)_{\omega, \epsilon}=( & -1)^{m} \frac{\left(-i \eta_{b}\right)_{m}}{m !} \lim _{\lambda \rightarrow 0}\left((1-\delta)^{-i \eta_{c}-1}\left(\frac{1-\delta}{1-d_{1}}\right)^{1+i \eta_{c}}(1-\gamma)^{i \eta_{a}}\left(\frac{1-f_{1}}{1-\gamma}\right)^{i \eta_{c}} \times\right. \\
& \times\left(1-f_{1}\right)^{-m} \sum_{n=0}^{\infty} \frac{\left(1+i \eta_{c}\right)_{n}\left(-i \eta_{a}\right)_{n}}{n ! n !}\left((-1)^{n}(1-\gamma)^{-n} \lambda^{n}\left(\frac{1-\delta}{1-d_{1}}\right)^{n} \times\right. \\
& \times F_{1}\left(1+i \eta_{b},-n, n+1+i \eta_{c}, m+1 ; \frac{\delta}{\delta-1}, \frac{d_{1}}{d_{1}-1}\right) \times \\
& \left.\left.\times F_{1}\left(1+i \eta_{a},-m, m-i \eta_{c}, n+1 ; \gamma, \frac{\gamma-f_{1}}{1-f_{1}}\right)\right)\right) .
\end{aligned}
$$

Найдем выражение, которое получается в результате предельных переходов в (48) и затем в формуле (36) для интеграла перекрытия. Используя соотношения (33)(35), получаем

$$
\begin{gathered}
f_{1} \simeq 1+u \epsilon, \quad b_{1} \simeq 1+v \epsilon, \quad d_{1} \simeq x+\epsilon d_{2}, \quad h_{1} \simeq-x+\epsilon h_{2}, \\
u=\frac{\left(c_{0}-f_{0}\right) \xi_{1}}{c_{0}}=\frac{c_{0}\left(c_{0}-f_{0}\right)}{f_{0} g_{0}+c_{0} h_{0}}, \quad v=\frac{\left(a_{0}-b_{0}\right) \xi_{1}}{a_{0}}=\frac{\left(a_{0}-b_{0}\right) c_{0}^{2}}{a_{0}\left(f_{0} g_{0}+c_{0} h_{0}\right)}, \\
x=\frac{c_{0} d_{0}-a_{0} g_{0}}{a_{0}\left(c_{0}-g_{0}\right)}, \quad d_{2}=\frac{c_{0}^{2}\left(d_{0}-a_{0}\right)}{a_{0}\left(c_{0}-g_{0}\right)^{2}}, \quad d_{2}+h_{2}=\left(-x-\frac{b_{0} g_{0}+c_{0} h_{0}}{a_{0}\left(c_{0}-g_{0}\right)}\right) \xi_{1} .
\end{gathered}
$$

5 Теоретическая и математическая физика, т. 146, № 2, 2006 г. 
Отсюда при $\epsilon \rightarrow 0$ для коэффициента при $\left(Y^{m}\right)_{\omega, \epsilon}$ в формуле (36) имеем

$$
(1-\alpha)^{i \eta_{b}-m}(1-\beta)^{i \eta_{a}} \epsilon^{m} \rightarrow \epsilon^{m-i \eta_{a}}\left(\frac{c_{0}-g_{0}}{c_{0}}\right)^{i \eta_{b}-m}\left(-\xi_{1}\right)^{-i \eta_{a}}
$$

Найдем результат предельного перехода при $\lambda \rightarrow 0$. Предельное выражение в (48) разбивается на произведение пределов с конечными значениями. Рассмотрим коэффициент перед знаком суммирования в правой части (48). После подстановки в него значений параметров согласно соотношениям (49) получаем

$$
\begin{aligned}
\lim _{\lambda \rightarrow 0}\left(\left(1-f_{1}\right)^{-m}(1-\delta)^{-i \eta_{c}-1}\left(\frac{1-\delta}{1-d_{1}}\right)^{1+i \eta_{c}}(1-\gamma)^{i \eta_{a}}\left(\frac{1-f_{1}}{1-\gamma}\right)^{i \eta_{c}}\right)= \\
=\frac{\epsilon^{i \eta_{a}-m}}{1-x-\epsilon d_{2}}\left(\left(\frac{u}{v}\right)^{i \eta_{c}}(-u)^{-m}(-v)^{i \eta_{a}}\left(-v x-v \epsilon d_{2}-d_{2}-h_{2}\right)^{-i \eta_{c}} \times\right. \\
\left.\quad \times\left(\frac{v x+v \epsilon d_{2}+d_{2}+h_{2}}{x+\epsilon d_{2}-1}\right)^{i \eta_{c}}\right)
\end{aligned}
$$

Далее определяем предел коэффициента перед функциями Аппеля в формуле (48). Учитывая (43), (49), находим

$$
\begin{aligned}
\lim _{\lambda \rightarrow 0} & \left((1-\gamma)^{-n} \lambda^{n}\left(\frac{1-\delta}{1-d_{1}}\right)^{n}\right)= \\
& =\lim _{\lambda \rightarrow 0}\left((-v \epsilon-\lambda)^{-n}\left(x+\epsilon d_{2}-1\right)^{-n}\left(v x \epsilon+(v \epsilon+\lambda+1) d_{2} \epsilon+h_{2} \epsilon+x \lambda-\lambda\right)^{n}\right)= \\
& =\left(\frac{v x+v \epsilon d_{2}+d_{2}+h_{2}}{v\left(-x-\epsilon d_{2}+1\right)}\right)^{n} .
\end{aligned}
$$

Подставляя в формулу (36) найденные предельные значения для ее компонентов, получаем для предельного значения произведения (51) и правой части (52) при $\epsilon \rightarrow 0$

$$
\begin{aligned}
\frac{1}{1-x}(- & \left.\frac{c_{0}}{u\left(c_{0}-g_{0}\right)}\right)^{m}\left(\left(\frac{u}{v}\right)^{i \eta_{c}}(-v)^{i \eta_{a}}\left(\frac{c_{0}-g_{0}}{c_{0}}\right)^{i \eta_{b}} \times\right. \\
& \left.\times\left(-v x-d_{2}-h_{2}\right)^{-i \eta_{c}}\left(-\frac{v x+d_{2}+h_{2}}{1-x}\right)^{i \eta_{c}}\left(-\xi_{1}\right)^{-i \eta_{a}}\right) .
\end{aligned}
$$

Так же легко вычисляется предельное значение коэффициента (53):

$$
\lim _{\epsilon \rightarrow 0}\left(\frac{v x+v \epsilon d_{2}+d_{2}+h_{2}}{v\left(-x-\epsilon d_{2}+1\right)}\right)^{n}=\left(\frac{v x+d_{2}+h_{2}}{v(1-x)}\right)^{n}
$$

Чтобы найти предельные значения функций Аппеля, определим предельные зна- 
чения их аргументов. Используя формулы (43), (49) и (50), находим

$$
\begin{aligned}
\frac{\delta}{\delta-1} & \rightarrow \lim _{\epsilon \rightarrow 0} \lim _{\lambda \rightarrow 0} \frac{\lambda}{\epsilon\left(v x+(v \epsilon+1) d_{2}+h_{2}\right)}+1=1, \\
\frac{d_{1}}{d_{1}-1} & \rightarrow \lim _{\epsilon \rightarrow 0} \frac{x+\epsilon d_{2}}{x+\epsilon d_{2}-1}=\frac{x}{x-1}=\frac{a_{0} g_{0}-c_{0} d_{0}}{c_{0}\left(a_{0}-d_{0}\right)}, \\
\gamma & \rightarrow \lim _{\epsilon \rightarrow 0} \lim _{\lambda \rightarrow 0}(v \epsilon+\lambda+1)=1, \\
\frac{\gamma-f_{1}}{1-f_{1}} & \rightarrow \lim _{\epsilon \rightarrow 0} \lim _{\lambda \rightarrow 0}\left(-\frac{-u \epsilon+v \epsilon+\lambda}{u \epsilon}\right)=\frac{u-v}{u}=\frac{b_{0} c_{0}-a_{0} f_{0}}{a_{0}\left(c_{0}-f_{0}\right)} .
\end{aligned}
$$

Таким образом, предельные значения первых аргументов в обеих функциях Аппеля обращаются в единицу. Поэтому для дальнейшего упрощения выражения (48) можно использовать формулу $(5.10(10))$ из книги [20]. Учитывая симметрию, очевидную из формулы (46), попарно меняем местами второй и третий параметры и аргументы в стандартной записи функций Аппеля. После этого функции Аппеля, фигурирующие в правой части (48), сводятся к гипергеометрическим функциям Гаусса согласно следующим равенствам:

$$
\begin{aligned}
F_{1}(1+ & \left.i \eta_{b}, n+1+i \eta_{c},-n, m+1 ; \frac{x}{x-1}, 1\right)= \\
= & \frac{\Gamma(m+1) \Gamma\left(m+n-i \eta_{b}\right)}{\Gamma\left(m-i \eta_{b}\right) \Gamma(m+n+1)} F\left(1+i \eta_{b}, n+1+i \eta_{c}, m+n+1 ; \frac{x}{x-1}\right)= \\
= & \frac{\left(-i \eta_{b}\right)_{m+n} m !}{\left(-i \eta_{b}\right)_{m}(m+n) !} F\left(1+i \eta_{b}, n+1+i \eta_{c}, m+n+1 ; \frac{a_{0} g_{0}-c_{0} d_{0}}{c_{0}\left(a_{0}-d_{0}\right)}\right), \\
F_{1}(1+ & \left.i \eta_{a}, m-i \eta_{c},-m, n+1 ; \frac{u-v}{u}, 1\right)=\frac{\Gamma(n+1) \Gamma\left(m+n-i \eta_{a}\right)}{\Gamma\left(n-i \eta_{a}\right) \Gamma(m+n+1)} \times \\
\quad & \left(\frac{v}{u}\right)^{-1-i \eta_{a}} F\left(1+i \eta_{a}, n+1+i \eta_{c}, m+n+1 ; \frac{v-u}{v}\right)=\frac{\left(-i \eta_{a}\right)_{m+n} n !}{\left(-i \eta_{a}\right)_{n}(m+n) !} \times \\
& \times\left(\frac{\left(a_{0}-b_{0}\right) c_{0}}{a_{0}\left(c_{0}-f_{0}\right)}\right)^{-1-i \eta_{a}} F\left(1+i \eta_{a}, n+1+i \eta_{c}, m+n+1 ; \frac{a_{0} f_{0}-b_{0} c_{0}}{\left(a_{0}-b_{0}\right) c_{0}}\right) .
\end{aligned}
$$

Для преобразования гипергеометрической функции в формуле (57) мы применили формулу $(2.1 .4(22))$ из книги [20].

Таким образом, в результате подстановки (48) в правую часть (36) и последовательного вычисления пределов получаем выражение, в котором под знаками суммирования будет стоять произведение выражений (54), (55), (56), (57). В это произведение входят отношения символов Похгаммера к подобным факториалам, две пары из которых взаимно сокращаются. После вынесения из-под знаков суммирования 
выражений, не зависящих от индексов суммирования, получаем

$$
\begin{aligned}
X_{0, \omega}= & \frac{4 \pi u}{v(1-x)}\left(a_{0}^{-1-i \eta_{c}} c_{0}^{i \eta_{c}}\left(\frac{u}{v}\right)^{i\left(\eta_{a}+\eta_{c}\right)}(-v)^{i \eta_{a}}\left(\frac{c_{0}-g_{0}}{c_{0}}\right)^{i \eta_{b}} \times\right. \\
& \times\left(-v x-d_{2}-h_{2}\right)^{-i \eta_{c}}\left(-\frac{v x+d_{2}+h_{2}}{1-x}\right)^{i \eta_{c}} \times \\
& \times\left(-\xi_{1}\right)^{-i \eta_{a}} \sum_{m=0}^{\infty}\left(\frac { ( - i \eta _ { c } ) _ { m } } { m ! } ( \frac { c _ { 0 } } { u ( c _ { 0 } - g _ { 0 } ) } ) ^ { m } \sum _ { n = 0 } ^ { \infty } \left(\frac{\left(1+i \eta_{c}\right)_{n}}{n !}\left(\frac{\left(-i \eta_{b}\right)_{m+n}}{(m+n) !}\right) \times\right.\right. \\
& \times\left(\frac{\left(-i \eta_{a}\right)_{m+n}}{(m+n) !}\right)\left(-\frac{v x+d_{2}+h_{2}}{v(1-x)}\right)^{n} \times \\
& \times F\left(1+i \eta_{b}, n+1+i \eta_{c}, m+n+1 ; \frac{x}{x-1}\right) \times \\
& \left.\left.\times F\left(1+i \eta_{a}, n+1+i \eta_{c}, m+n+1 ; \frac{v-u}{v}\right)\right)\right)
\end{aligned}
$$

Используя соотношения (50), находим зависимость коэффициентов и параметров, фигурирующих в (58), от параметров, определенных соотношениями в формуле (26). Для замены аргументов гипергеометрических функций используем формулы (56) и (57). Выполняя в (58) суммирование по множеству пар целых чисел, заполняющих первый квадрант, заменим суммирование по полуоси $m$ суммированием по диагонали $m+n \rightarrow m$. В результате получаем представление для интеграла перекрытия:

$$
\begin{aligned}
& X_{0, \omega}=K_{0}(\omega) \sum_{m=0}^{\infty}\left(\frac{\left(-i \eta_{b}\right)_{m}\left(-i \eta_{a}\right)_{m}}{m ! m !} \sum_{n=0}^{m} \frac{\left(-i \eta_{c}\right)_{m-n}\left(1+i \eta_{c}\right)_{n}}{(m-n) ! n !}(U(\omega))^{m-n}(V(\omega))^{n} \times\right. \\
& \left.\quad \times F\left(1+i \eta_{b}, n+1+i \eta_{c}, m+1 ; x(\omega)\right) F\left(1+i \eta_{a}, n+1+i \eta_{c}, m+1 ; y(\omega)\right)\right) .
\end{aligned}
$$

Функции, зависящие от параметра $\omega$, определяются выражениями

$$
\begin{gathered}
U(\omega)=\frac{f_{0} g_{0}+c_{0} h_{0}}{\left(c_{0}-f_{0}\right)\left(c_{0}-g_{0}\right)}, \quad V(\omega)=\frac{b_{0} d_{0}+a_{0} h_{0}}{\left(a_{0}-b_{0}\right)\left(a_{0}-d_{0}\right)}, \\
x(\omega)=\frac{a_{0} g_{0}-c_{0} d_{0}}{c_{0}\left(a_{0}-d_{0}\right)}, \quad y(\omega)=\frac{a_{0} f_{0}-b_{0} c_{0}}{c_{0}\left(a_{0}-b_{0}\right)}, \\
K_{0}(\omega)=4 \pi a_{0}^{-1-i \eta_{c}} c_{0}^{i \eta_{c}} \frac{a_{0}\left(c_{0}-g_{0}\right)}{c_{0}\left(a_{0}-d_{0}\right)}\left(\frac{a_{0}\left(c_{0}-f_{0}\right)}{c_{0}\left(a_{0}-b_{0}\right)}\right)^{1+i\left(\eta_{a}+\eta_{c}\right)}\left(1-\frac{g_{0}}{c_{0}}\right)^{i \eta_{b}} \times \\
\times\left(-\frac{c_{0}^{2}}{f_{0} g_{0}+c_{0} h_{0}}\right)^{-i \eta_{a}}\left(-\frac{\left(a_{0}-b_{0}\right) c_{0}^{2}}{a_{0}\left(f_{0} g_{0}+c_{0} h_{0}\right)}\right)^{i \eta_{a}} \times c^{i \eta_{c}}\left(\frac{c_{0}^{3}\left(b_{0} d_{0}+a_{0} h_{0}\right)}{a_{0}^{2}\left(c_{0}-g_{0}\right)\left(f_{0} g_{0}+c_{0} h_{0}\right)}\right)^{-i \eta_{c}} .
\end{gathered}
$$

Для дальнейшего упрощения коэффициента $K_{0}(\omega)$ необходимо определить численные значения фаз оснований степенны́х функций, фигурирующих в последней из 
формул (60). Численные расчеты были выполнены при $\omega=10^{-17} \Phi_{\mathrm{M}^{-1}}$ на примере развала ядер ${ }^{6} \mathrm{Li}$ на ядрах ${ }^{208} \mathrm{~Pb}$ при энергии $156 \mathrm{MэВ.} \mathrm{Для} \mathrm{вычисления} \mathrm{парамет-}$ ров, определенных формулами (26), использовались соотношения нерелятивистской трехчастичной кинематики [21]. Далее были выполнены такие преобразования: если фаза основания оказывалась близкой к $\pm \pi$, то из степенной функции выносился коэффициент $e^{ \pm i \pi x}$, где $x$ - показатель степенно́й функции. В результате такой процедуры комплексные значения оснований всех степеней перемещаются в правую полуплоскость. Оказывается, что фазу, близкую к $-\pi$, имеют только $c_{0}$ и пара оснований степеней с показателями $\pm i \eta_{a}$, вклады которых взаимно компенсируются. Остается только вклад множителя $e^{\pi \eta_{c}}$, который определяет большие значения (порядка $10^{9}-10^{12} \Phi_{\mathrm{M}^{-2}}$ ) коэффициента $\left|K_{0}(\omega)\right|$, а с ними и заметную величину кулоновского развала на две заряженные частицы. В результате получено следующее выражение:

$$
K_{0}(\omega)=\frac{4 \pi\left(c_{0}-g_{0}\right)^{1+i \eta_{c}}}{c_{0}^{2+i \eta_{c}}}\left(\frac{a_{0}\left(c_{0}-f_{0}\right)}{\left(a_{0}-b_{0}\right)\left(a_{0}-d_{0}\right)}\right)^{1+i \eta_{c}}\left(1-\frac{f_{0}}{c_{0}}\right)^{i \eta_{a}}\left(1-\frac{g_{0}}{c_{0}}\right)^{i \eta_{b}} .
$$

Вычисление производной согласно формуле (16) для матричного элемента $T_{f i(\omega)}$ является технической задачей, которая решается с помощью математических пакетов Mathematica, Maple и других. В конечной формуле можно положить $\omega=0$ в параметрах $U, V, x, y$, а также в их (логарифмических для $U(\omega), V(\omega))$ производных. Тогда $U, V, x, y$ будут действительными. Однако при использовании пакета Mathematica выполнение полного контрольного расчета для случая кулоновского развала ядер лития оказалось невозможным. Оказалось, что пакет не вычисляет необходимых гипергеометрических функций при $m>170$. Поэтому программа была составлена в пакете Maple. Для вычисления гипергеометрических функций в алгоритме используются рекуррентные формулы вниз по $m$ и вверх по $n$ с коррекцией значений гипергеометрических функций через 20-30 шагов по $n$. Недавно нами был выполнен расчет трижды дифференциальных сечений кулоновского развала ядер ${ }^{3} \mathrm{He}$, рассеивающихся на ядрах ${ }^{90} \mathrm{Zr}$ при энергии $90 \mathrm{MэВ} \mathrm{[22].}$

\section{4. ПРЕДСТАВЛЕНИЕ ИНТЕГРАЛА ПЕРЕКРЫТИЯ ЧЕРЕЗ КОНТУРНЫЙ ИНТЕГРАЛ}

Более просто можно получить альтернативное представление интеграла перекрытия (17), а именно в виде однократного интеграла по контуру в комплексной плоскости. Вернемся к формуле (27). В подынтегральном выражении интеграла по $t$ введем обозначения

$$
A=a_{0}-s d_{0}, \quad B=b_{0}+s h_{0} .
$$

Далее в результате дробно-линейного преобразования переменной интегрирования

$$
t=\frac{A \tau}{A-B+B \tau}
$$


внутренний интеграл сводится к интегральному представлению гипергеометрической функции Гаусса, а сам интеграл перекрытия приводится к выражению

$$
\begin{array}{rl}
X_{0, \omega}=2 & i\left(d_{0}^{-1-i \eta_{a}-i \eta_{c}} g_{0}^{i \eta_{c}}\left(d_{0}+h_{0}\right)^{i \eta_{a}} \int_{1}^{(0+)}(1-s)^{i \eta_{b}}(-s)^{-1-i \eta_{b}} \times\right. \\
& \left.\times\left(s_{1}-s\right)^{-1-i\left(\eta_{a}+\eta_{c}\right)}\left(s_{2}-s\right)^{i \eta_{c}}\left(s_{3}-s\right)^{i \eta_{a}} F\left(-i \eta_{c},-i \eta_{a}, 1 ; y_{0}(s)\right) d s\right),
\end{array}
$$

где

$$
\begin{gathered}
y_{0}(s)=\frac{f_{1} s^{2}+b_{1} s+a_{1}}{\left(s_{2}-s\right)\left(s_{3}-s\right)}, \\
a_{1}=\frac{a_{0} f_{0}-b_{0} c_{0}}{g_{0}\left(d_{0}+h_{0}\right)}, \quad b_{1}=\frac{-d_{0} f_{0}+b_{0} g_{0}+\left(a_{0}-c_{0}\right) h_{0}}{g_{0}\left(d_{0}+h_{0}\right)}, \\
f_{1}=\frac{\left(g_{0}-d_{0}\right) h_{0}}{g_{0}\left(d_{0}+h_{0}\right)}, \quad s_{1}=\frac{a_{0}}{d_{0}}, \quad s_{2}=\frac{c_{0}}{g_{0}}, \quad s_{3}=\frac{a_{0}-b_{0}}{d_{0}+h_{0}} .
\end{gathered}
$$

В интеграле (61) можно избавиться от сингулярности в особой точке $s_{1}$, если перенести ее на бесконечность посредством дробно-линейного преобразования

$$
s=\frac{s_{1} t}{s_{1}+t-1} .
$$

Тогда интеграл перекрытия преобразуется к форме

$$
\begin{array}{rl}
X_{0, \omega}=2 & i K_{2,(\omega)} \int_{1}^{(0+)}(1-t)^{i \eta_{b}}(-t)^{-1-i \eta_{b}}\left(t-t_{2}\right)^{i \eta_{c}}\left(t_{3}-t\right)^{i \eta_{a}} \times \\
& \times F\left(-i \eta_{c},-i \eta_{a}, 1 ; x_{0}(t)\right) d t .
\end{array}
$$

Аргумент $x_{0}(t)$ и параметры, присутствующие в этом выражении, определяются следующими соотношениями:

$$
\begin{gathered}
x_{0}(t)=\frac{t^{2}+b_{2} t+a_{2}}{\left(t_{2}-t\right)\left(t_{3}-t\right)}, \quad a_{2}=\frac{\left(a_{0}-d_{0}\right)^{2}\left(a_{0} f_{0}-b_{0} c_{0}\right)}{\left(a_{0} g_{0}-c_{0} d_{0}\right)\left(b_{0} d_{0}+a_{0} h_{0}\right)}, \\
b_{2}=\frac{\left(a_{0}-d_{0}\right)\left(b_{0}\left(a_{0} g_{0}-2 c_{0} d_{0}\right)+a_{0}\left(d_{0} f_{0}+\left(a_{0}-c_{0}\right) h_{0}\right)\right)}{\left(a_{0} g_{0}-c_{0} d_{0}\right)\left(b_{0} d_{0}+a_{0} h_{0}\right)}, \\
t_{2}=\frac{c_{0}\left(a_{0}-d_{0}\right)}{a_{0} g_{0}-c_{0} d_{0}}, \quad t_{3}=\frac{\left(a_{0}-b_{0}\right)\left(a_{0}-d_{0}\right)}{b_{0} d_{0}+a_{0} h_{0}}, \\
K_{2,(\omega)}=e^{\pi \eta_{c}} a_{0}^{-1}\left(\frac{a_{0}-d_{0}}{a_{0}}\right)^{i \eta_{b}}\left(\frac{a_{0} g_{0}-c_{0} d_{0}}{a_{0}\left(a_{0}-d_{0}\right)}\right)^{i \eta_{c}}\left(\frac{b_{0} d_{0}+a_{0} h_{0}}{a_{0}\left(a_{0}-d_{0}\right)}\right)^{i \eta_{a}} .
\end{gathered}
$$

Если выбрать $\omega=10^{-17} \Phi_{M}^{-1}$, то особые точки $t_{2}$ и $t_{3}$ будут лежать практически на действительной оси. Проведенные расчеты показали, что особая точка $t_{2}<0$, а точка $t_{3}>1$. С ростом энергии частицы $b$ особые точки сначала приближаются (правда, не одновременно) к пределам интегрирования, а затем удаляются от них. Наименьшие расстояния между точками $t=0 \quad(t=1)$ и этими особыми точками составляют несколько сотых. Это значит, что в окрестности точек $t=0$ 
и $t=1$ при некоторых значениях энергии частицы $b$ подынтегральная функция будет осциллировать. Последнее может понизить точность вычисления. Однако выполнение тождественных преобразований (см. [20], (2.1.4(22))) гипергеометрической функции под интегралом невозможно, поскольку при этом появляется особая точка, значения которой сильно зависят от энергий вылетающих частиц и могут быть как внутри, так и вне интервала интегрирования. Таким образом, вычисления надо производить с повышенной точностью, которая должна обеспечиваться программными средствами. Особенность в точке $t=1$ можно проинтегрировать по малому промежутку после аппроксимации подынтегральной функции отрезком степенно́го ряда. Гипергеометрическая функция вычисляется между узлами интегрирования путем параллельного интегрирования дифференциального уравнения для этой функции методом Рунге-Кутта.

\section{5. ЗАКЛЮЧЕНИЕ}

В работе представлены два метода вычисления амплитуды кулоновского развала на два заряженных кластера в приближении нулевого радиуса взаимодействия между кластерами. Выражение интеграла перекрытия $X_{0, \omega}$ через двукратный ряд (59) в области сходимости этого ряда является тождественным представлением интеграла в формуле (62). Это можно проверить на частном случае, положив $\eta_{a}=0$. Другой вид интегрального представления, аналогичного (62), можно получить после перестановки порядка интегрирования в формуле $(27)$ и переобозначения $t \leftrightarrow s$ переменных интегрирования. Тогда для проверки надо положить $\eta_{b}=0$.

Метод суммирования двукратного ряда можно использовать также для определения поправок первого порядка на конечность радиуса взаимодействия между кластерами, поскольку эти поправки содержат градиенты по импульсам частиц от найденного выше интеграла перекрытия. Интегралы перекрытия, с помощью которых выражаются поправки второго порядка, содержат комбинации нулевых, первых или вторых производных от функций Куммера вылетающих частиц. Поэтому они могут быть сведены только к представлениям через контурные интегралы. Наиболее сложным оказывается вычисление контурного интеграла, в котором вместо функции Гаусса стоит функция Аппеля двух переменных. В этом случае надо наряду с интегрированием решать задачу Коши для системы двух уравнений (5.9 (9)) из книги [20] в частных производных для функции Аппеля.

Благодарности. Автор выражает благодарность профессору Д. Траутману за предоставленную распечатку одного из вариантов программы BREAK4 и присланную работу.

\section{Список литературы}

[1] V. Corcalcius, H. Jelitto. Coincidence cross sections within the quasi free break-up model for elastic projectile break-up. Preprint KfK 4960. Karlsruhe: Institut für Kernphysik, Kernforschungszentrum Karlsruhe GmbH, 1991.

[2] R. Serber. Phys. Rev. 1947. V. 72. P. 1008. 
[3] E.H.L. Aarts, R. Malpliet, S.Y. van der Werf, R.J. de Meijer. Nucl. Phys. A. 1982. V. 380. № 3. P. 465.

[4] А.Г. Ситенко, А.Д. Полозов, М.В. Евланов. УФЖ. 1974. Т. 19. №11. С. 1778; А.Г. Ситенко, М.В. Евланов, А.Д. Полозов, А.М. Соколов. ЯФ. 1986. Т. 43. № 1. C. $78 ;$ 1987. T. 45. № 5. C. 1320; N. Heide, H. Rebel, V. Corcalcius et al. Elastic break-up of $156 \mathrm{MeV}{ }^{6} \mathrm{Li}$ projectile with large asymptotic relative momenta of the fragments. Preprint KfK 4564. Karlsruhe: Institut für Kernphysik, Kernforschungszentrum Karlsruhe GmbH, 1989; M.В. Евланов, А. М. Соколов, B.К. Тартаковский. ЯФ. 1996. Т. 59. № 4. С. 679; 2003. Т. 66. № 2. С. 278.

[5] F. Rybicki, N. Austern. Phys. Rev. C. 1972. V. 6. P. 1525.

[6] H. Rebel, D.K. Srivastava. Mechanisms of Li-projectile break-up. Preprint KfK 4761. Karlsruhe: Institut für Kernphysik, Kernforschungszentrum Karlsruhe GmbH, 1991.

[7] G. Baur, D. Trautmann. Phys. Rep. 1976. V. 25. P. 293.

[8] Л.Д. Ландау, Е. М. Лифиии. ЖЭТФ. 1948. Т. 18. № 8. С. 750.

[9] A. Goto, H. Kamitsubo, N. Matsuoka, H. Sakaguchi. Nucl. Phys. A. 1985. V. 444. P. 248.

[10] M. Kawai. Progr. Theor. Phys. Suppl. 1986. V. 89. P. 11.

[11] Y. Iseri, M. Yahiro, M. Kamimura. Progr. Theor. Phys. Suppl. 1986. V. 89. P. 84; Y. Sakuragi, M. Yahiro, M. Kamimura. Progr. Theor. Phys. Suppl. 1986. V. 89. P. 136 .

[12] R. Shyam, G. Baur, F. Rösel, D. Trautmann. Phys. Rev. C. 1980. V. 22. P. 1401

[13] C.M. Vincent, H. T. Fortune. Phys. Rev. C. 1970. V. 2. P. 782.

[14] К.О. Теренецкий. ЯФ. 1983. Т. 37. № 5. С. 1177; А.П. Ильин, К.О. Теренецкий. Изв. АН СССР. Сер. физ. 1984. Т. 48. № 2. С. 359; А.П. Ильин. Адиабатическое представление задачи трех тел в теории рассеяния слабосвязанных легких ядер. Автореферат канд. дисс. Киев: Институт ядерных исследований НАН Украины, 1995.

[15] М. Абрамович, И. Стиган. Справочник по специальным функциям. М.: Наука, 1979.

[16] Е. О. Альт, Б. Ф. Иргазиев, А.Т. Муминов, А.М. Мухамеджанов. ЯФ. 1995. Т. 58. № 11. C. 1967.

[17] Г. М. Фихтенгольи. Курс дифференциального и интегрального исчисления. Т. 2. М.: Наука, 1966

[18] Л.Д. Ландау, Е. М. Лифшиц. Квантовая механика. Нерелятивистская теория. М.: Физматгиз, 1963

[19] Е. Янке, Ф. Эмде, Ф. Леш. Специальные функции. М.: Наука, 1968.

[20] Г. Бейтмен, А. Эрдейи. Высшие трансцендентные функции. Т. І. Гипергеометрическая функция. Функции Лежандра. М.: Наука, 1973.

[21] G. G. Ohlsen. Nucl. Instr. Meth. 1965. V. 37. P. 240.

[22] A.P. Ilyin. ${ }^{90} \mathrm{Zr}\left({ }^{3} \mathrm{He}, d p\right)^{90} \mathrm{Zr}$ at $E_{L}=90 \mathrm{MeV}$. In: LV National Meeting on Nuclear Spectroscopy and Nuclear Structure "Frontiers in the Physics of Nucleus" (St.Petersburg, Russia, 28 June -1 July 2005). Book of abstracts. St.Petersburg: St.Petersburg State University, 2005. P. 244. 